\title{
Effect of Oxidative Stress on Iraqi Rheumatoid Arthritis Patients
}

\author{
Weaam F. Hussain and Wisam Kadhum H. Al-Hashemi \\ Department of Chemistry, College of Science, Al-Nahrain University, Baghdad-Iraq. \\ Corresponding Author: weam911@yahoo.com.
}

\begin{abstract}
Oxidative stress is an imbalance between oxidants and antioxidants, play an important role in the development of Rheumatoid Arthritis. Rheumatoid Arthritis regard as a chronic, autoimmune disease. The aim of the study to assess levels of some oxidative stress biomarker in Rheumatoid Arthritis. This study involves 80 Rheumatoid arthritis patients and 30 healthy subjects as control groups. Analysis on serum samples was Malondialdehyde, Albumin, and total Bilirubin. The obtained data showed significantly increasing $(\mathrm{P}<0.01)$ in level of oxidant parameter Malondialdehyde and significantly decrease $(\mathrm{P}<0.01)$ in endogenous antioxidant albumin and total bilirubin in comparison with the control group which may be due to increased activity of immune system which involves reactive oxygen species in its action. The reactive oxygen species of originated from the immune system have the crucial role in oxidative stress situation in Rheumatoid arthritis. [DOI: $10.22401 /$ JNUS.21.2.04]
\end{abstract}

Keywords: Oxidative stress, Rheumatoid arthritis, Malondialdehyde.

\section{Introduction}

Rheumatoid arthritis (RA) is a chronic, autoimmune and progressive inflammatory disease, which exhibit inflammatory responses through of participation of inflammatory cells [1], which attacks synovial joints that leads to the cartilage and bone erosion [2]. The main etiology of disease is unknown, but may be developed with an environmental component like smoking, microbiota [3]. The characterized of RA affects approximately 0.5$1 \%$ of the population of world [4] and affects in women more than men [5] but it's unknown the etiology.

Immune system reacts with endogenous or exogenous antigen which produces oxidant in situ. Therefore it's the main participant in causing and deterioration RA symptoms. Oxidant plays an important role in tissue injure in RA [1]. Overproduction of reactive oxygen species (ROS) leads to damage of proteins, lipids, nucleic acids, and matrix components of joint in this disease. [6]. ROS as oxidant is highly reactive species that include species such as Superoxide $\left({ }^{\circ} \mathrm{O}_{2}{ }^{\circ}\right)$ and Hydroxyl $\left({ }^{\circ} \mathrm{OH}\right)$, while the other type of Oxidant reactive nitrogen species (RNS) that Include species such as Nitric oxide ( $\mathrm{NO}^{\circ}$ ) and Nitrogen dioxide $\left(\mathrm{NO}_{2}{ }^{\circ}\right)$. Those species Participate to promote inflammation of RA joint patients [7].

ROS has two sources: Endogenous (mitochondria, peroxisomes, phagocytic cells etc.) and exogenous (Environmental pollution, bacteria, and viruses). Endogenous sources are important and extensive than exogenous sources because these sources are a continuous process during the life span of every cell in the organism. But both exogenous and endogenous sources produce ROS as byproduct during metabolism in vivo. [8,9] Which lead to oxidative stress situation (Imbalance between ROS and Antioxidant).

Superoxide radical has a long half-life and highly reactive species which produced in the mitochondrial membrane ROS. The enzymes could produce superoxide which includes xanthine oxidase, lipooxygenase, cyclooxygenase, and NADPH dependent oxidase. [8,9]

Hypochlorous Acid ( $\mathrm{HOCl})$ is non-free radical generated by activation of neutrophils at the site of inflammation. As result of reaction hydrogen peroxide with chloride ion by catalyzed the enzyme myeloperoxidase, and that produces $\mathrm{HOCl}$.[10].

$$
\mathrm{H}_{2} \mathrm{O}_{2}+\mathrm{Cl}^{-} \rightarrow \mathrm{HOCl}+\mathrm{OH}
$$

Oxidative stress contributes with RA progression, it take place when immune cells activate in synovial membrane or cavity. Immune cells transfer from blood vessels into the inflammatory area, which lead to increase excretion of inflammatory mediators in RA that include ROS, RNS. [1,10].

Biomarker of oxidative stress in RA is 
divided into two parts oxidant and antioxidant. Oxidant such as Malondialdehyde (MDA). MDA play role exceeds becoming a lipid peroxidation byproduct because its intensify inflammation by producing immunogenic molecule and causing cell damage. However, loss of lubrication of synovial fluid is evidence of exposure the synovial fluid to superoxide and hydrogen peroxide.[11] Elevated serum MDA reported in RA patients [12, 11, 13].

Antioxidants are molecules have the ability to neutralize oxidants and prevent cells damage, Antioxidant can be classified in two groups: exogenous and endogenous Antioxidant.[14]

Endogenous Antioxidant such as Albumin, and Total bilirubin. Albumin regard as major antioxidant in plasma[16], which has activities related to ligand-binding capacities. [15].

Total bilirubin is a powerful antioxidant that could prevent lipid oxidation and other kinds of oxidation [16] total Bilirubin with regard to RA risk plays protective effect by getting rid of peroxyl radicals [28].

\section{Materials and Methods}

This study involved 80 patients RA (11 male 69 females) with matching control group 30 volunteers (10 males and 20 females) age between (20-69) years.it had done in Kadhimiya and Yarmouk Hospitals in the

Table (1)

Characteristics of the Rheumatoid arthritis and control group (mean \pm SEM).

\begin{tabular}{|c||c||c|c||}
\hline Characteristic & RA patient group $N=80$ & Control group $N=30$ & P value \\
\hline \hline Age $($ year) & $45.2 \pm 1.3$ & $37.8 \pm 2.5$ & $<0.05$ \\
\hline \hline BMI kg/m & $28.3 \pm 0.57$ & $26.5 \pm 0.73$ & $<0.05$ \\
\hline RF IU/mL & $11.55 \pm 1.24$ & $<8$ & --------- \\
\hline MDA $\mu \mathrm{mol} / \mathrm{L}$ & $8.13 \pm 0.24$ & $3.82 \pm 0.17$ & $<0.01$ \\
\hline \hline Albumin $\mathrm{g} / \mathrm{dL}$ & $3.86 \pm 0.1$ & $4.75 \pm 0.13$ & $<0.01$ \\
\hline Total bilirubin $\mathrm{mg} / \mathrm{dL}$ & $0.33 \pm 0.12$ & $0.41 \pm 0.04$ & $<0.05$ \\
\hline
\end{tabular}

The high level of oxidants in RA patients promote oxidative stress situation which manifested as an increase of lipid peroxidation marker MDA and at comparison with control group. [11]

The high level of MDA may be due to excessive oxidant concentration which promotes by activation of the immune system as rheumatoid factor indicates (see table above). The excessive oxidant came from cells period from November 2016 to February 2017. All patients diagnosed by a physician with an exclusion of diseases that have oxidative stress such as cancer and diabetes as well as smokers. The biomarkers used in this study were; Serum MDA (done according to Shah S.V. \& Walker method) [17], serum albumin supplied by (Spinreact, Spain)[18] and serum total bilirubin (kit supplied by Biomaghreb) [19].

\section{Statistics analysis}

In this study all statistical analysis used SPSS program. To descriptive analysis was used to show the mean and standard error mean (SEM). by using Student's T-test. The figures were done by used Prizam7 program. Difference consider significantly at $(p<0.05)$. [20]

\section{Results and Discussion}

Table (1) shows the means and standard error mean of age, body mass index (BMI), Rheumatoid factor (RF), malondialdehyde (MDA), albumin and total bilirubin for the control and patients groups.

The results revealed that patients with RA had an almost two and a half times higher levels of serum MDA than control group as shown in Table (1). of the immune system in synovial fluid leads to an intense oxidative stress condition. And to the same reason lead to formation hypoxiareperfusion so increase oxidative stress as result increase of MDA concentration [1, 21]. As shown in the Fig.(1) these result agreements with several studies [11, 22, 10].

In opposite to oxidant, endogenous antioxidant showed significant decrease in its levels manifested in significant decrease of 
albumin level in compare to control group, as shown in the Table (1).

Albumin is known to be one of the negative acute phase proteins [23] and it is also found to have antioxidant properties [24]. So the decrease in serum albumin level obtained in the present study could be explained by either that albumin is one of the negative acute phase proteins that decreased after inflammations or due to its activity as antioxidant or both, as shown in the Fig.(2) The results obtained in the present study agree with [25] [26].

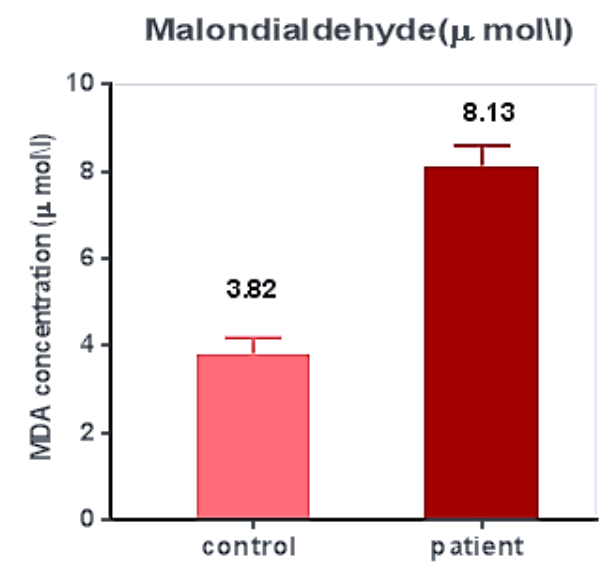

Fig.(1): Serum MDA concentration ( $\mu \mathrm{mol} / \mathrm{l})$ in $R A$ at comparison with control group.

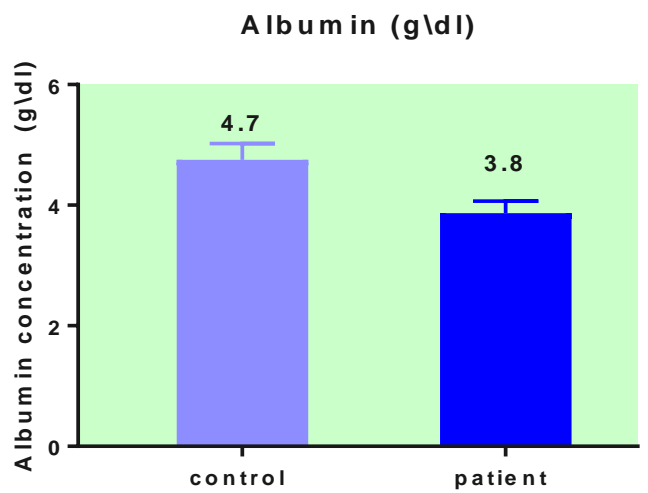

\section{Fig.(2): Serum albumin concentration ( $g \backslash d l)$ in $R A$ at comparison with control group.}

Serum Total bilirubin levels is significant decrease in compare to control group, as shown in the Fig.(3).

Bilirubin is a molecule with effective antioxidant properties due to its structure, therefore, it to exhibit its antioxidant properties that lead to the consumption of this molecules and decreasing its levels in the serum of RA patients. Since RA is an inflammatory disease produces more free radicals more than normal. Total bilirubin acts as a protective factor for RA risk, it gets rid of peroxyl radicals and decreases oxidative stress [27]. This study agreement with studies [27,28].

MDA and endogenous antioxidant (albumin and total bilirubin) have no correlation between them this may be due to the ratio of deterioration in antioxidant as a result of its activity toward the oxidants haven't direct proportion with oxidation by-product (i.e. MDA). This observation may be due to the antioxidant activity was the direct product of its reaction with oxidant while the oxidation byproduct generation was secondary product to the reaction of oxidant with the biomolecules.

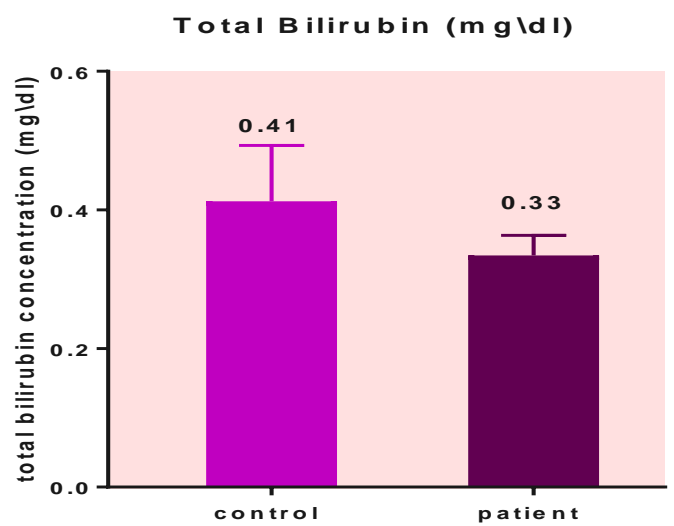

Fig.(3): Serum Total bilirubin concentration ( $m g \backslash d l)$ in RA at comparison with control group.

\section{Conclusion}

The significant increase in oxidants and in parallel the significant decrease in endogenous antioxidants clarify the oxidative stress role as cause an exacerbation factor for rheumatoid arthritis disease.

\section{Acknowledgements}

The authors would like to thank AlNahrain University for research facilities.

\section{References}

[1] Asis B., Chaitali M., Pallab K., "Oxidative stress in inflammatory cells of patient with rheumatoid arthritis: clinical efficacy of dietary antioxidants." Inflammopharmacology $1-13,2017$.

[2] Arata N,. Yasuchika A., "Identification of clinical parameters associated with serum oxidative stress in patients with rheumatoid 
arthritis." Modern rheumatology. 24(6), 926930, 2014.

[3] Angelotti F., Parma A., "One year in review 2017: pathogenesis of rheumatoid arthritis." Clin Exp Rheumatol 35, 368378, 2017.

[4] Ishibashi, Toru, "Consumption of water containing a high concentration of molecular hydrogen reduces oxidative stress and disease activity in patients with rheumatoid arthritis: an open-label pilot study." Medical gas research 2(1), 27, 2012.

[5] Somaiya M., Shagufta M., Atif Z.,. "Redox signaling in rheumatoid arthritis and the preventive role of polyphenols." Clinica Chimica Acta 463, 4-10, 2016.

[6] Taibur R, Ismail H, "Oxidative stress and human health." Advances in Bioscience and Biotechnology 3, 997-1019, 2012.

[7] Adolfo G., Ramón Gaxiola-Robles, and Tania Z., "Oxidative stress in patients with rheumatoid arthritis." Rev Invest Clin 67(1), 46-53, 2015.

[8] Kohen R, and Abraham N. "Invited review: Oxidation of biological systems: oxidative stress phenomena, antioxidants, redox reactions, and methods for their quantification." Toxicologic pathology 30 (6),620-650, 2002.

[9] Phaniendra A., Dinesh B., and Latha P., "Free radicals: properties, sources, targets, and their implication in various diseases." Indian Journal of Clinical Biochemistry 30 (1), 11-26, 2015.

[10] Datta S., "Correlation of oxidant status with oxidative tissue damage in patients with rheumatoid arthritis." Clinical rheumatology 33(11) 1557-1564, 2014.

[11] Chandankhede, M. S., and M. M. Gupta. "Oxidative stress and antioxidant status in patients with rheumatoid arthritis." Int J Biol Med Res 4 (2), 3088-3090, 2013.

[12] Quiñonez F., Celia M., "Oxidative stress relevance in the pathogenesis of the rheumatoid arthritis: a systematic review." BioMed research international, 1-14, 2016.

[13] Kamanlı A., "Plasma lipid peroxidation and antioxidant levels in patients with rheumatoid arthritis." Cell biochemistry and function 22.(1) 53-57, 2004.

[14] Pramod J., Sheena S., and Joydeep Singh. "Role of free radicals and antioxidants in human health and disease." International Journal of Current Research and Review 5
(19), 14, 2013.

[15] Bourdon E., and Denis B,. "The importance of proteins in defense against oxidation." Antioxidants and Redox Signaling 3 (2), 293-311, 2001.

[16] Batta A., "Bilirubin as savior of biological system.” Int. J. Curr. Res. Med. Sci 3 (2), 21-27, 2017.

[17] Shah S.V. \&Walker P.D. "Evidence suggesting a role for hydroxyl radical in glycerol-induced acute renal failure". Am. J. Physiol. 255, F438, 1988.

[18] Young D., and Richard B. "Friedman. Effects of disease on clinical laboratory tests. Amer Assn for Clinical Chemistry", 2001.

[19] walters M.1., Gerarde R.W., Microchem. 15, 23, 1970.

[20] McDonald, John H. Handbook of biological statistics. Baltimore, MD: Sparky House Publishing, 3, 2013.

[21] Łuczaj W., "The onset of lipid peroxidation in rheumatoid arthritis: consequences and monitoring." Free radical research 50(3), 304-313, 2016.

[22] Vyas S., Hemlata S., and Vyas RK. "Role of malondialedehed in the serum of Rheumatoid arthritis and osteoarthritis." Journal of Postgraduate Medical Institute 30 (1), 2016.

[23] Quinlan Gregory J., Greg S. Martin, and Timothy W. Evans. "Albumin: biochemical properties and therapeutic potential." Hepatology 41 (6), 1211-1219, 2005.

[24] Roche M., "The antioxidant properties of serum albumin." FEBS letters 582 (13) 1783-1787, 2008.

[25] Sahebari M., "Serum trace element concentrations in rheumatoid arthritis." Biological trace element research 171(2), 237-245, 2016.

[26] Kowsalya, R., "Protein carbonyl content and thiols in patients with rheumatoid arthritis." Biomedicine 31(4), 555-557, 2011.

[27] Fischman, D., "Bilirubin as a protective factor for rheumatoid arthritis: an NHANES study of 2003-2006 data." Journal of clinical medicine research 2(6), 256, 2010.

[28] Juping, Du, "Serum bilirubin and the risk of rheumatoid arthritis." Journal of Clinical Laboratory Analysis, e22118, 1-6, 2017. 